\title{
Analyse du régime alimentaire de l'ichtyofaune dans les étangs piscicoles traditionnels de la Basse Casamance (Sénégal)
}

Ngor NDOUR1*, Bienvenu SAMBOU2, Ngansoumana BA2 Yacinthe SAMBOU2 et Maurice DASYLVA1 1 Université Assane SECK de Ziguinchor, Département d'Agroforesterie, Sénégal.

2 Université Cheikh Anta Diop de Dakar, Sénégal

* Auteur correspondant ; E-mail : ngorndour@univ-zig.sn ; BP : 523, Tél : + 2215533203

Original submitted in on 11th September 2017. Published online at www.m.elewa.org on 30 th November 2017 https://dx.doi.org/10.4314/jab.v119i1.3

\section{RÉSUMÉ}

Objectif : Cette étude vise à connaître le régime alimentaire des poissons élevés dans les étangs piscicoles en Basse-Casamance. Méthodes et résultats : Afin de fournir des informations sur la nourriture des poissons élevés, des prélèvements d'eau des étangs et de contenus stomacaux des poissons récoltés, ont été analysés de juillet 2014 à mars 2016. La présence et l'abondance de phytoplanctons, dans ces prélèvements, ont montré l'existence d'un réseau trophique constitué d'une chaîne alimentaire planctophage et d'une autre détritivore. La première montre une similarité de régime alimentaire chez Sarotherodon melanotheron (Tilapia à gorge noire) aussi bien à Bandial qu'à Mlomp. Une autre similarité de régime planctophage est notée entre Sarotherodon melanotheron, Tilapia guineensis (Tilapia de guinée) et le groupe des mulets qui se nourrissent en partie de Gyrosigma atenuatum dans les deux contrées. Le régime alimentaire détritivore est noté chez Ethmalosa fimbriata (Etnmalose) et Hemichromis bimaculatus (Cichlidé joyaux à deux taches). Un régime alimentaire omnivore est noté chez Hemichromis fasciatus (Hemichromis rayé) qui se nourrit à la fois de détritus végétaux et de larves d'insectes comme c'est le cas chez les fretins du groupe des mulets. En définitive, la taille des principales espèces de poisson à la récolte laisse penser que la productivité naturelle des étangs, associée aux nutriments charriés par les eaux de ruissellement, suffit à nourrir convenablement les poissons. Toutefois, un apport alimentaire pourrait booster la croissance de certaines espèces de poissons notamment Pomadasys jubelini (Carpe blanche), Solea senegalensis (sole du Sénégal) et Sphyraena barracuda (Barracudas) à Bandial. Une telle mesure pourrait certainement se traduire par une production de poissons de meilleure qualité chez Mugil cephalus (Mulet cabot), Liza grandisquamis (Mulet à grandes écailles), L. falcipinis (Mulet à grandes nageoires), Elops Lacerta (Guinée du Sénégal), Tilapia guineensis, Sarotherodon melanotheron et Hemichromis fasciatus. La productivité naturelle des étangs indique que la pisciculture extensive peut servir de moteur de développement de l'aquaculture au Sénégal. L'exploitation du savoir local a permis de fabriqué un aliment en cours de test dans les bassins piscicoles du département d'agroforesterie.

Mots clés : détritivore, étangs poissons, phytoplancton, régime alimentaire, similarité. 
Diet analysis of fishes in traditional ponds at low-Casamance (Senegal).

\begin{abstract}
Objective: This study aims at knowing the diet of breed-raised fish in ponds in Lower Casamance.

Methods and Results: To provide information on the diet of breed-raised fish, water samples of ponds and stomach contents of collected fishes were analyzed from July, 2014 to March, 2016. The presence and abundance of phytoplankton, in these samples, showed the existence of a trophic network consisting of a planktophagous food chain and a detritivore food chain. The first shows a similarity of diet for Sarotherodon melanotheron (Blackchin tilapia) in Bandial and Mlomp. Another similarity of a planktophagous diet is noted between Sarotherodon melanotheron, Tilapia guineensis (Guinean tilapia), and the group of mules which feed partly on Gyrosigma atenuatum in both regions. The detritivore diet is noted for Ethmalosa fimbriata and Hemichromis bimaculatus (Jewelfish). An omnivorous diet is noted for Hemichromis fasciatus which feeds both on plant detritus and insect larvae. Ultimately, the size of the main fish species harvested suggests that the natural productivity of ponds, coupled with nutrients transported by runoff water, is sufficient to feed the fish properly. However, a food intake could boost the growth of some fish species including Pomadasys jubelini (Sompat grunt), Solea senegalensis (lascar) and Sphyraena barracuda (Great barracuda). Such a measure could certainly lead to a high quality fish production for Mugil cephalus (Grey mullet), Liza grandisquamis (Largescaled Mullet), L. falcipinis (Sicklefin mullet), Elops Lacerta (West African ladyfish), Tilapia guineensis Sarotherodon melanotheron and Hemichromis fasciatus (Banded jewelfish). The natural productivity of the ponds indicates that the extensive fish breeding can be used as engine of development of aquiculture in Senegal. The exploitation of the local knowledge allowed of manufactured a food in the course of test in the piscicultural basins of the agroforestry department.
\end{abstract}

Keywords: diet, ponds, fish, phytoplankton, scavenger, similarity

\section{INTRODUCTION}

La pisciculture est en déclin en Basse Casamance au Sénégal à cause de la détérioration des systèmes hydro-agricoles ancestraux (idée Casamance, 2007, Bassene, 2016). D'ailleurs, le nombre d'étangs a diminué dans la zone et la pisciculture intégrée aux formations de mangrove s'est raréfiée (Bambara, 1989). Le système d'exploitation piscicole est caractérisé par des polders qui protègent les rizières contre les eaux salées du bolon. Ces polders délimitent des bassins utilisés traditionnellement pour la pisciculture (Idée-Casamance, 2007). Ils sont construits et entaillés dans la zone qui sépare l'étang $\mathrm{du}$ cours d'eau. Ces entailles permettent aux poissons à la recherche de sites de ponte de regagner les étangs à la bonne période (mai-juin). C'est ainsi que les géniteurs se regroupent pendant la marée haute à proximité des entailles de la digue qui permettent aux pisciculteurs d'empoisonner et d'élever des poissons dans les unités de production sans apport alimentaire. Ce savoir-faire s'amenuise alors que les besoins en poisson augmentent du fait de la croissance démographique (Bassene et al.,
2013 ; ANSD, 2015). II s'y ajoute un manque de main d'œuvre qui paralyse le renouvellement des digues. Néanmoins, certains pisciculteurs ont maintenu ce système de production sans se soucier de la nourriture disponible et de sa quantité dans les étangs. II convient donc de connaître les aliments qui permettent le fonctionnement des exploitations traditionnelles. A cet égard, la présente étude s'intéresse au régime alimentaire des poissons. L'abondance des aliments suffit-elle à expliquer la réussite de la pisciculture traditionnelle? Pour répondre à cette question, cette étude a porté sur l'analyse du bol alimentaire des poissons et des eaux les étangs de Bandial et de Mlomp (Figure 1). En tout état de cause, les enquêtes réalisées sur le régime alimentaire des poissons ont permis de noter que les producteurs connaissent en partie les aliments des poisons élevés dans les étangs notamment ceux de Wawdjugué, Amind'éwole et Yambathine à Bandial et ceux de Mlomp : Jaman, Entienne $_{1}$ et Etienne 2 , Albert 1 et Albert 2. 


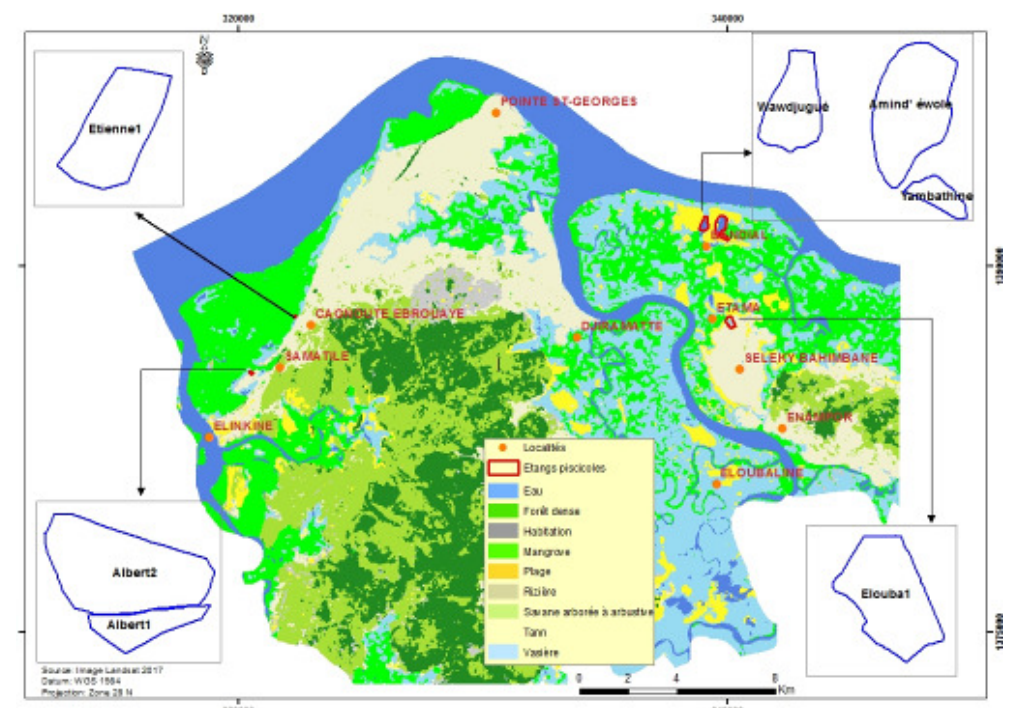

Figure 1 : Localisation des étangs piscicoles en Basse Casamance

\section{MATÉRIEL ET MÉTHODES}

Les échantillons d'eau sont prélevés le long du plus grand axe de l'étang, à la surface, à mi- profondeur et au fond des étangs (Figure 2). Périodiquement, la collecte se fait à l'empoisonnement, à mi- élevage et pendant la récolte des poissons. Un échantillon composite est élaboré par niveau de prélèvement et traité au formol à $3,4 \%$. Les analyses réalisées au laboratoire ont permis d'identifier les microorganismes présents dans les étangs à différentes phases du cycle d'élevage.

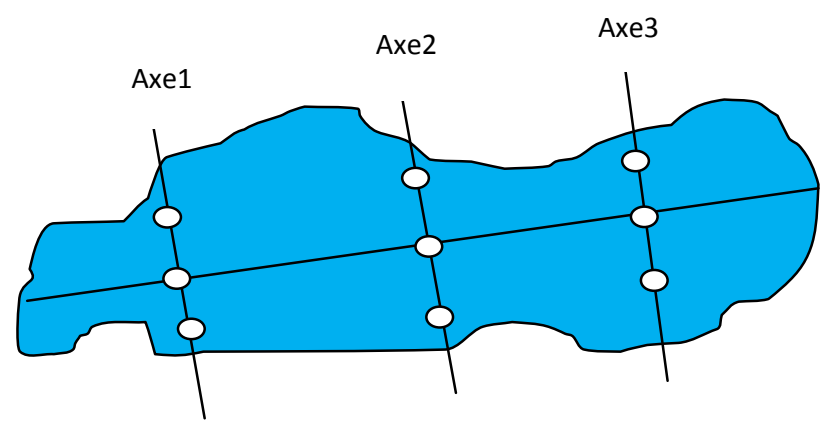

Figure 2 : dispositif de prélèvement des échantillons

Au cours de la récolte, les poissons sont recensés, puis des spécimens prélevés et éviscérés au laboratoire. Ainsi, le contenu stomacal de poissons de la même espèce a servi à l'élaboration d'un échantillon également traité au formol à $3,4 \%$ afin de conserver la forme des microorganismes dans l'échantillon. Ces derniers ont été identifiés à l'aide d'un microscope au laboratoire de botanique et de biodiversité végétale de l'Université Cheikh Anta Diop de Dakar. L'analyse du contenu stomacal des poissons a permis de noter la présence ou l'absence de phytoplanctons ou zooplanctons dans le suc stomacal des poissons. A cet égard, le suc stomacal de
357 individus du groupe des mulets a été examiné, celui de 656 individus de Sarotherodon melanotheron, un autre de 50 individus de Tilapia guineensis, un de 27 individus de Elops lacerta et enfin un de 03 individus de Hemichromis fasciatus pêchés dans les étangs de Bandial. A Mlomp, 23 individus de Tilapia guineensis et 10 individus de mulets ont servi à l'analyse du suc stomacal des poissons. Afin de montrer une similarité de régime alimentaire entre les poissons dans les étangs, une analyse factorielle de correspondance (AFC) a été réalisée. Elle a permis de montrer une similarité de régime alimentaire au sein de la même espèce dans des 
étangs différents et une autre entre des espèces différentes dans le même étang ou dans des étangs différents. La deuxième approche méthodologique a porté sur l'analyse de la perception de la nourriture des poissons par les pisciculteurs à l'aide d'un questionnaire. Les informations recueillies auprès des producteurs sont fondées sur leur sens de l'observation et leur connaissance empirique de la pisciculture. A cet effet, 74 producteurs ont été interrogés dans la zone d'étude dont 21 dans la contrée de Bandial et 53 dans celle de Mlomp. L'observation, du comportement des poissons dans les étangs, a aussi été utilisée pour la collecte de données complémentaire sur leur régime alimentaire. Elle a consisté à observer les poissons dans leur milieu de vie

\section{RÉSULTATS}

Diversité des espèces de poissons élevés dans les étangs de la zone d'étude : Dans la zone, 16 espèces de poissons sont rencontrées à Bandial contre 10 espèces à Mlomp (Tableau 1). La famille des Cichlidae et celle des Mugilidae y sont plus représentées. La classe des Cichlidae est représentée par 4 espèces de poissons dont Sarotherodon melanotheron, Tilapia guineensis, pendant deux heures de temps à mi- élevage et pendant la récolte. A cet égard, des herbacées aquatiques consommées par les poissons ont été identifiées dans les étangs de Mlomp.

Traitement des données : Les prélèvements d'eau et de sucs stomacaux des poissons sont analysés à l'aide d'un microscope. La similarité de régime alimentaire des poissons a été analysée avec xISATpro7.5 sur la base d'une AFC. L'abondance moyenne des microorganismes est calculée par étang et par site de prélèvement à l'aide du progiciel Excel. Les données d'enquêtes ont été recueillies à l'aide du logiciel Cspro. 6 puis traitées à l'aide du logiciel SPSS.

Hemichromis fasciatus et $H$. bimaculatus. Celle des Mugilidae est constituée de Mugil cephalus, M. curema (mulet blanc), Liza falcipinis, $L$. bananensis et $L$. grandisquamis. A Bandial, Elops lacerta (Elopideae) est bien représentée lors de la récolte aussi bien à Wawdjugué qu'à Amind'éwole.

Tableau 1: Espèces de poissons élevées par contrée dans la zone d'étude

\begin{tabular}{|l|l|c|c|}
\hline Espèces de poissons & Familles & Bandial & Mlomp \\
\hline Sarotherodon melanotheron Rüppel, 1852 & Cichlidae & + & + \\
\hline Tilapia guineensis Bleeker, 1862 & Cichlidae & + & + \\
\hline Hemichromis fasciatus Peters, 1857 & Cichlidae & + & + \\
\hline Hemichromois bimaculatus Gill, 1862 & Cichlidae & + & + \\
\hline Mugil cephalus Linnaeus 1858 & Mugilidae & + & + \\
\hline Mugil curema & Mugilidae & + & + \\
\hline Liza falcipinis Valenciennes, 1836 & Mugilidae & + & + \\
\hline Liza grandisquamis Valenciennes, 1836 & Mugilidae & + & + \\
\hline Liza bananensis Pellgrin 1927 & Mugilidae & + & + \\
\hline Pomadasys jubelini Cuvier 1830 & Haemulidae & + & \\
\hline Elops lacerta C.V., 1830 & Elopidae & + & + \\
\hline Thorogobius angolensis Norman, 1935 & Gobiidae & + & \\
\hline Trachinotus teraia Cuvier et Valenciennes, 1832 & Carangidae & + & \\
\hline Ethmalosa fimbriata Bowdich ,1825 & clupeidae & + & \\
\hline Sphyraena barracuda Edwards, 1771 & Muranesocidae & + & \\
\hline Solea senegalensis Kaup, 1856 & Opisthoproctidae & + & \\
\hline
\end{tabular}

Abondance et diversité du phytoplancton dans les eaux des étangs piscicoles: L'abondance du phytoplancton évaluée dans les eaux et dans le suc stomacal des poissons, a varié entre 32000 et 512000 individus/ml dans la zone. Considérant sa diversité, la contrée de Bandial compte plus d'espèces (94 espèces) que celle de Mlomp (44) avec 29 espèces communes aux deux contrées.

Abondance et diversité du phytoplancton dans les étangs de Bandial: A Amind'éwole, l'abondance du phytoplancton variait entre 32000 et 160000 individus $/ \mathrm{ml}$ à l'empoisonnement. A la récolte, cette abondance a été de 32000 individus $/ \mathrm{ml}$. II ressort une baisse de 
Ndour et al., J. Appl. Biosci. 2017 Analyse du régime alimentaire de l'ichtyofaune dans les étangs piscicoles traditionnels de la Basse Casamance (Sénégal)

l'abondance entre l'empoisonnement et la récolte des poissons (Tableau 2, Figure 3). Les phytoplanctons sont

plus abondants et plus diversifiés à l'empoisonnement qu'à la récolte des poissons.

Tableau 2 : Abondance du phytoplancton

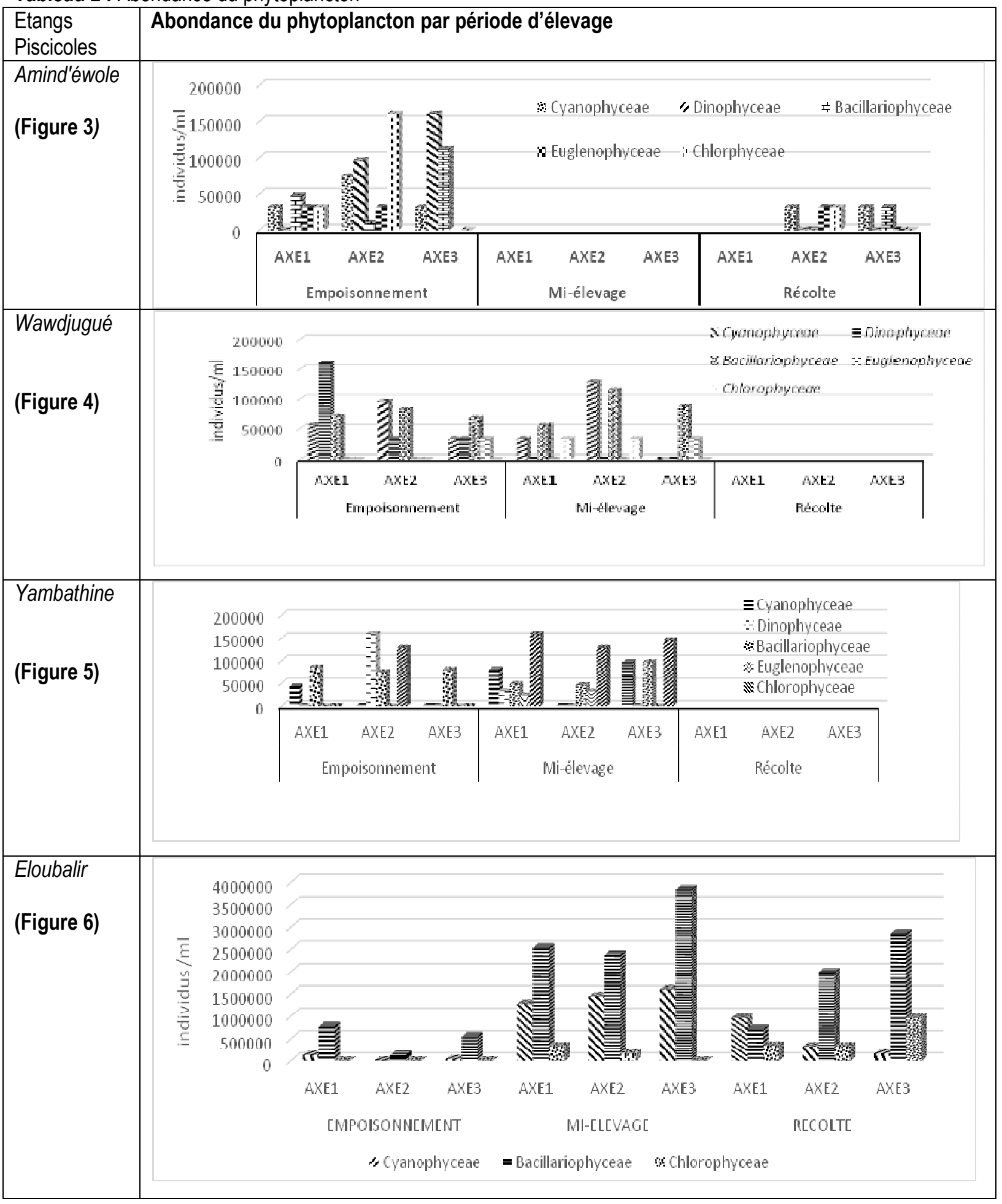



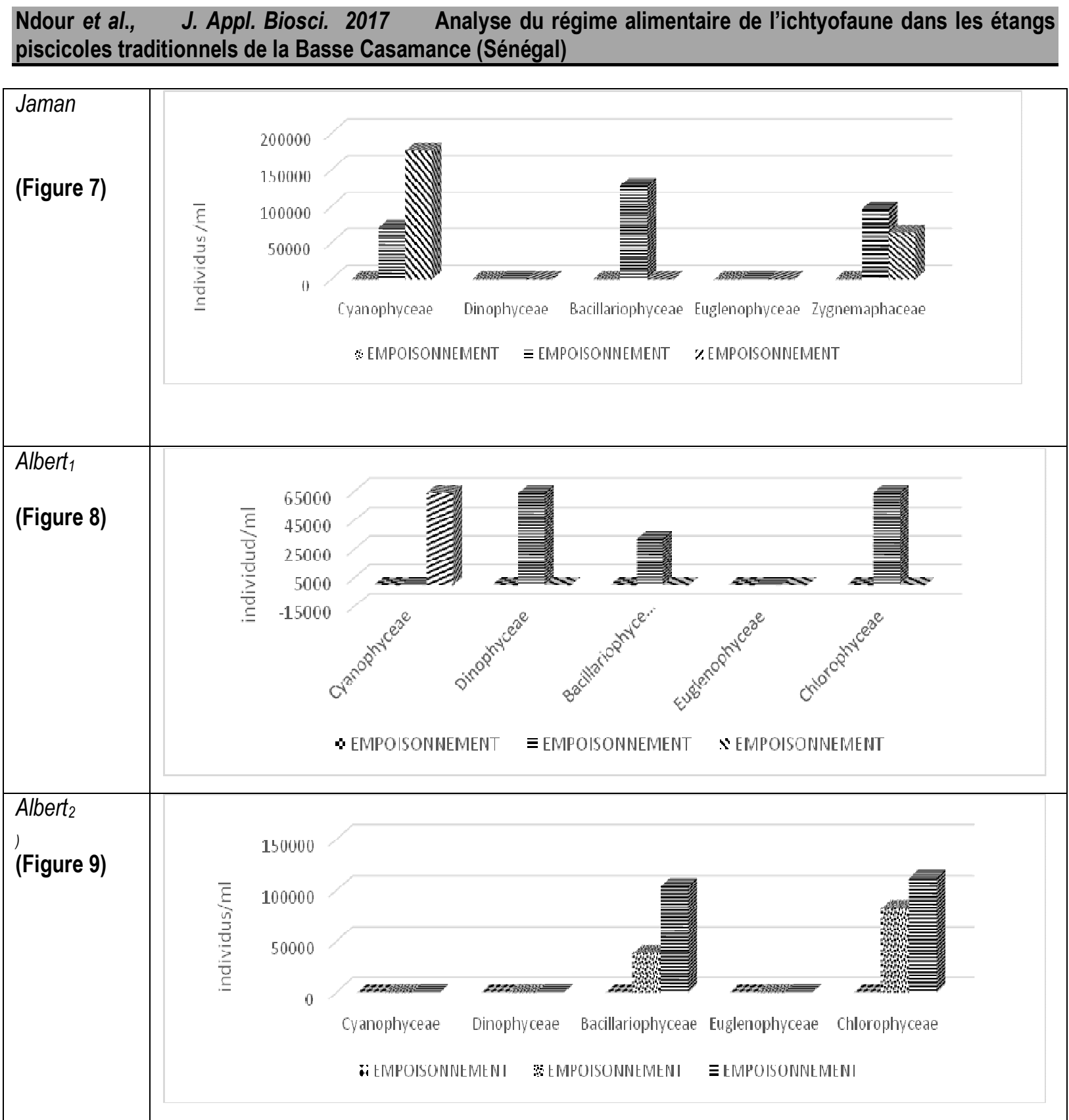

A Wawdjugué, cinq classes de phytoplancton sont représentées à l'empoisonnement. La classe des Dinophyceae est la plus abondante (Tableau 2, Figure 4). Parmi ces classes, les Euglenophyceae, Chlorophyceae, Bacillariophyceae, et Cyanophyceae sont encore présentes à mi- élevage avec une abondance plus importante à l'empoisonnement. A mi- élevage, la classe des Chlorophyceae vient de paraître dans l'étang avec une abondance moins importante que celle des autres phytoplanctons. Au cours de la récolte, la vidange de l'étang nuitamment n'a pas permis de prélever des échantillons d'eau. A Yambathine, les classes des Cyanophycae, Bacillariophyceae, Dynophyceae et
Chlorophyceae sont présentes à l'empoisonnement avec une abondance comprise entre 42666,66 et 160000 individus/ml (Tableau 2, Figure 5). A mi- élevage, la classe des Euglenophyceae apparait alors que celle des Dinophyceae a baissé en abondance par rapport à l'empoisonnement. A Eloubalir, la classe des Cyanophyceae présente une abondance relativement faible à l'empoisonnement (Tableau 2, Figure 6). L'abondance de cette classe augmente à mi- élevage et baisse pendant la récolte. Malgré cette baisse, l'abondance reste encore plus élevée à l'empoisonnement. La classe des Bacillariophyceae présente à l'empoisonnement devient plus abondante à 
mi- élevage, ce qui demeure jusqu'à la récolte. Les Chlorophyceae (320000-338000 individus/ml) apparaissent à mi- élevage et demeurent abondantes de l'empoissonnement jusqu'à la récolte.

Abondance et diversité du phytoplancton dans les étangs de Mlomp: A Jaman, les classes des Cyanophyceae, Bacillariophyceae et Zygnematophyceae présentent une abondance de phytoplancton comprise entre 64000 et 176000 individus/ml (Tableau 2, Figure 7). L'axe 1 de l'étang ne présente pas de phytoplanctons à l'empoisonnement. Cependant, les axes de prélèvements 2 et 3 présentent des phytoplanctons abondants. La proximité entre ces axes et les rizières, semble déterminer la présence et l'abondance du phytoplancton à l'empoisonnement. A Albert, les Cyanophyceae, les Dinophyceae, les Bacillariophyceae et les Chlorophyceae ont une abondance comprise entre 32000 et 64000 individus/ml à l'empoisonnement (Tableau 2, Figure 8). L'axe 1 de l'étang qui ne présente pas de phytoplanctons à l'empoissonnement est plus proche des eaux salée du bolon de Elinkine. A Albert, les Bacillariophyceae et les Chlorophyceae sont d'une abondance comprise entre 38400 et 112000 individus $/ \mathrm{ml}$ à l'empoisonnement (Tableau 2, Figure 9). Bien que l'étang ne soit pas diversifié en phytoplanctons leur abondance est relativement élevée. Les Cyanophyceae, les Dinophyceae, les Bacillariophyceae, les Chlorophyceae et les Euglenophyceae sont absentes de l'axe 1 à l'empoisonnement de l'étang. A cet égard, il convient de noter l'éloignement entre les étangs et le village de Samatite.

Régime alimentaire des poissons élevés dans les étangs piscicoles : L'étude du régime alimentaire des poissons, basée en partie sur l'analyse de leur suc stomacal et des eaux des étangs, a permis de relever les microorganismes consommés par les poissons à Bandial (Figure 10).

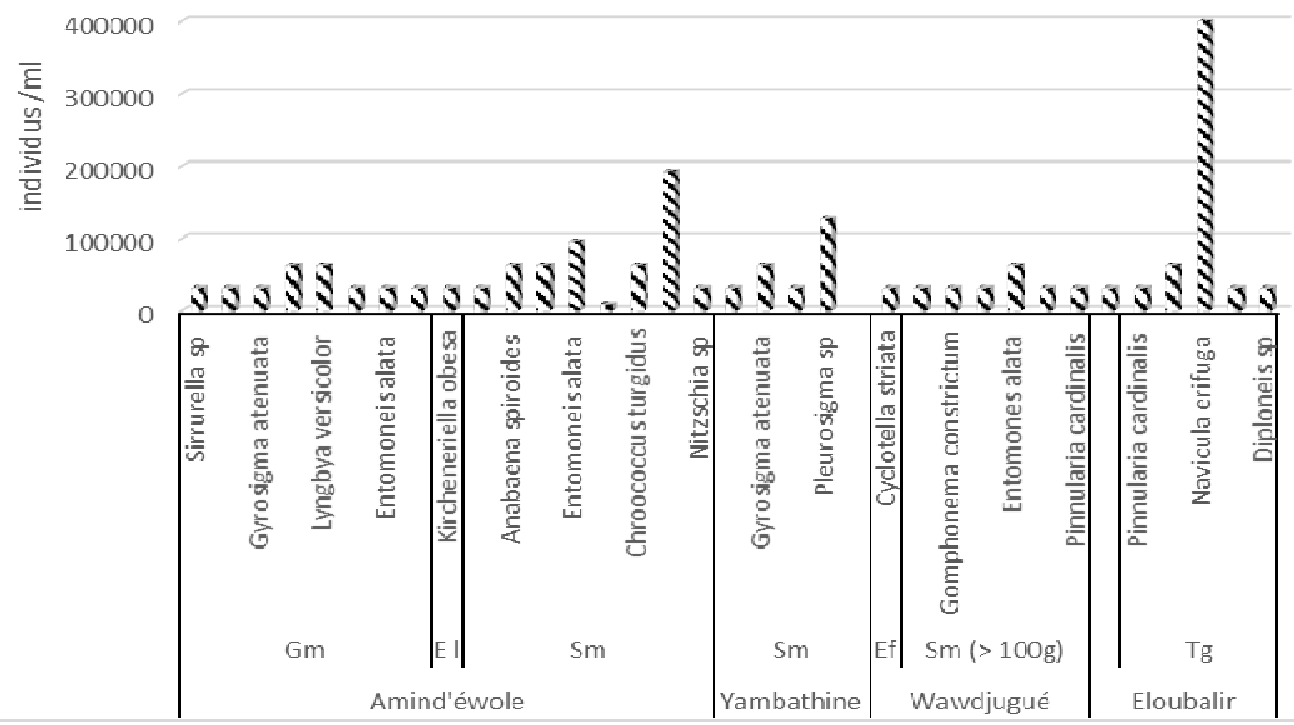

$\mathrm{Gm}=$ groupe des mulets; $\mathrm{El}=$ Elops lacerta; $\mathrm{Sm}=$ Sarotherodon melanotheron; $\mathrm{Ef}=$ Ethmalosa fimbrita; $\mathrm{Tg}=$ Tilapia guineensis . Figure 10: Abondance du phytoplancton consommé par les poissons à Bandial

Diversité du phytoplancton dans le bol alimentaire des poissons de Bandial: A Amind'éwole, le suc stomacal des poissons indique dans la figure 9 la présence de phytoplanctons abondants (32000 et 64000 individus / $\mathrm{ml}$ ) chez le groupe des mulets (Mugil cephalus, M. curema, Liza grandisquamis, $L$. bananensis et $L$. falcipinis). Chez Elops lacerta, le suc stomacal présente une abondance de 32000 individus $/ \mathrm{ml}$ de Kircheneriella obesa. Chez Sarotherodon melanotheron 4 phytoplanctons sont rencontrés à des concentrations comprises entre 64000 et 192000 individus/ml (Figure 10.) A Yambathine, l'abondance du Phytoplancton dans le suc stomacal de Sarotherodon melanotheron est comprise entre 32000 et 128000 individus $/ \mathrm{ml}$. Gyrosigma atenuatum et Pleurosigma sp sont les microorganismes notés. A Wawdjugué, le suc stomacal de Ethmalosa fimbriata contient des individus de Cyclotella striata. Celui de Sarotherodon melanotheron regorge de Gomphonema 
constrictum, Gyrosigma atenuatum, Entomoneis alata et Pinnularia cardinalis.

Diversité du phytoplancton dans le contenu stomacal des poissons de Mlomp : A Jaman, le suc stomacal de Sarotherodon melanotheron contient Entomoneis gigantea et Nitzschia acicularis (Figure 11). Chez le groupe des mulets deux phytoplanctons sont rencontrés notamment Navicula placentula et Pinnularia cardinalis. Chez Tilapia guineensis la gamme de phytoplanctons consommés est la plus large (Figure 11).

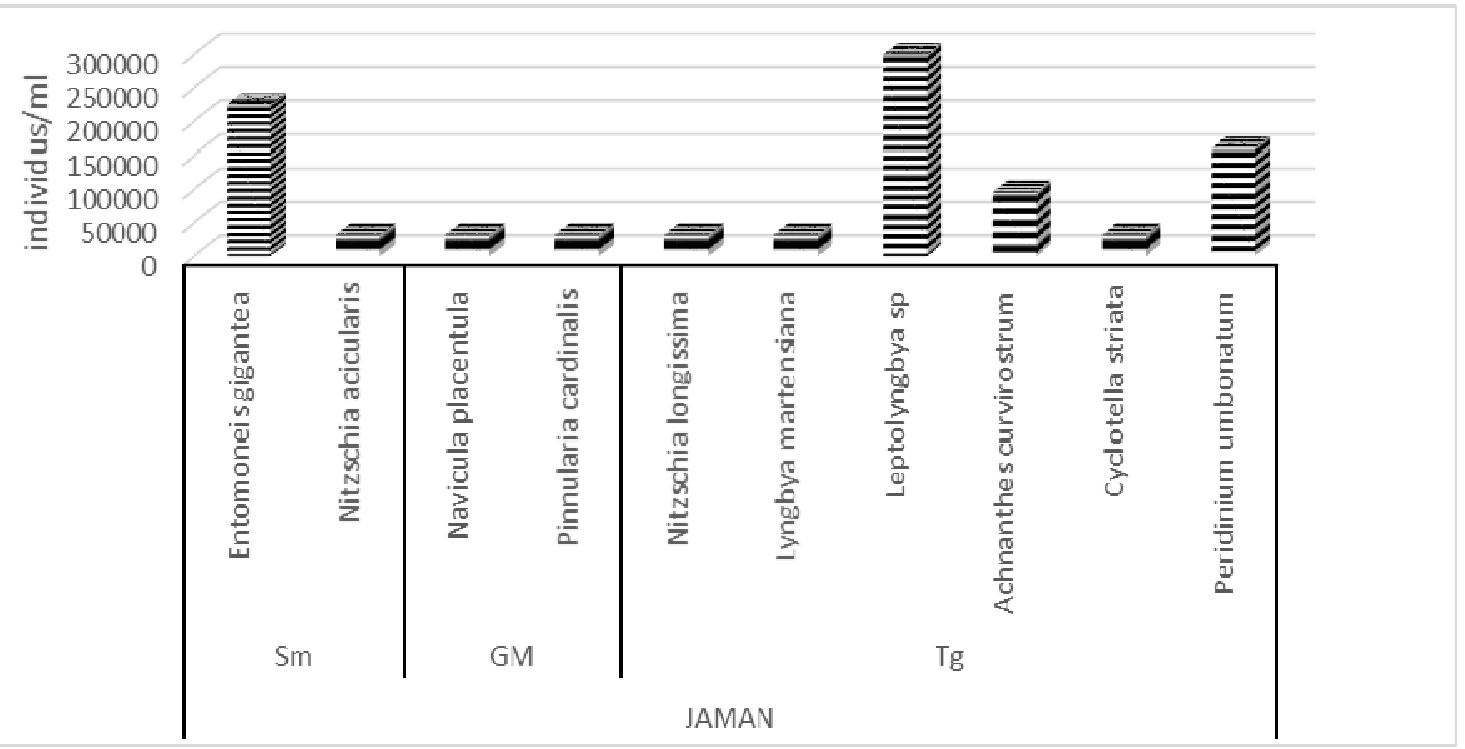

$\mathrm{Sm}=$ Sarotherodon melanotheron ; $\mathrm{GM}=$ groupe des mulets $; \mathrm{Tg}=$ Tilapia guinenensis

Figure 11 : Abondance du phytoplancton consommé par les poissons dans les étangs de Mlomp

Diversité du phytoplancton dans les étangs et le suc stomacal des poissons: A l'empoisonnement, les étangs de Bandial sont plus diversifiés en phytoplanctons que ceux de Mlomp. $33,33 \%$ des espèces de phytoplancton notées dans les eaux des étangs sont rencontrées dans le suc stomacal de cinq espèces de poissons (Sarotherodon melanotheron, Tilapia guineensis, Elops lacerta, Ethmalosa fimbriata et le groupe des mulets). Parmi ces espèces de phytoplancton $7,89 \%$ (Cyclotella striata, Gyrosigma atenuatum et Pinnularia cardinalis) servent à la fois de nourriture à Tilapia guineensis, Ethmalosa fimbriata et aux mulets. Cependant, les individus de Tilapia guineensis de taille moyenne $5 \mathrm{~cm}$, consomment des détritus végétaux à Mlomp. Considérant le régime alimentaire par espèce de poisson, Sarotherodon melanotheron a le régime alimentaire planctophage le plus diversifié avec 19 espèces consommées soit $16,81 \%$ du phytoplancton présent dans les étangs piscicoles. Les individus de Tilapia guineensis et le groupe des mulets viennent en deuxième position avec 12 espèces de phytoplancton soit $10,61 \%$ pour chacune des espèces de poisson. Ethmalosa fimbriata et Elops lacerta sont les poissons ayant le régime alimentaire planctophage le moins diversifié avec chacune une espèce de phytoplancton. Les poissons dont le suc stomacal ne présente pas de phytoplanctons sont constitués de Pomadasys jubelini, Thorogobius angolensis, Hemichromis bimaculatus et Hemichromis fasciatus. Dans le suc stomacal de ces poissons, il y'a des pattes d'insectes chez Pomadasys jubelini et des détritus végétaux chez les autres espèces. Analyse du régime alimentaire des poissons dans la zone d'étude: Le croisement du répertoire des phytoplanctons présents dans les étangs et celui des phytoplanctons présents dans le suc stomacal des poissons est consigné dans Tableau 3. 
Tableau 3: Régime alimentaire planctophage des poissons élevés dans la zone d'étude

\begin{tabular}{|c|c|c|c|c|c|c|c|c|c|c|}
\hline Espèces de phytoplanctons & Classes & $\mathrm{Sm}$ & $T g$ & $H f$ & $G m$ & $P j$ & El & $\mathrm{Ta}$ & Ef & $H b$ \\
\hline Achanthescurvirostrum & Bacillariophyceae & & $x$ & & & & & & & \\
\hline Anabaena spiroides & Cyanophyceae & $x$ & & & & & & & & \\
\hline Aphanocapsa halsatica & Cyanophyceae & $x$ & & & & & & & & \\
\hline Aphanocapsa sp & Cyanophyceae & $x$ & & & & & & & & \\
\hline Chlorophyceae versicolor & Cyanophyceae & & & & $x$ & & & & & \\
\hline Chroococcus turgidus & Cyanophyceae & $\mathbf{x}$ & & & & & & & & \\
\hline Closterium littorale & Chlorophyceae & $x$ & & & & & & & & \\
\hline Coconeis scutellum & Bacillariophyceae & $\mathbf{x}$ & & & & & & & & \\
\hline Coscinodiscus sp & Bacillariophyceae & & & & $\mathbf{x}$ & & & & & \\
\hline Cosmarium sp & Chlorophyceae & $x$ & & & & & & & & \\
\hline Cyclotella striata & Bacillariophyceae & & $\mathbf{x}$ & & $\mathbf{x}$ & & & & $\mathbf{x}$ & \\
\hline Cymbella cymbiformis & Bacillariophyceae & & & & & & & & & \\
\hline Diploneis sp & Bacillariophyceae & & $\mathbf{x}$ & & & & & & & \\
\hline Entomoneis alata & Bacillariophyceae & $\mathbf{x}$ & & & $\mathbf{x}$ & & & & & \\
\hline Entomoneis gigantea & Bacillariophyceae & $x$ & & & & & & & & \\
\hline Gloeocapsa sp. & Cyanophyceae & & & & $\mathbf{x}$ & & & & & \\
\hline Gomphoneis acuminatum var turris & Bacillariophyceae & & & & & & & & & \\
\hline Gomphonema constrictum & Bacillariophyceae & $x$ & & & & & & & & \\
\hline Gyrosigma atenuatum & Bacillariophyceae & $x$ & $\mathrm{x}$ & & $\mathrm{x}$ & & & & & \\
\hline Kircheneriella obesa & Chlorophyceae & & & & & & $\mathbf{x}$ & & & \\
\hline Leptolyngbya sp & Cyanophyceae & & $x$ & & & & & & & \\
\hline Lyngbya martensiana & Cyanophyceae & & $x$ & & & & & & & \\
\hline Lyngbya versicolor & Cyanophyceae & & $\mathbf{x}$ & & & & & & & \\
\hline Mastogloia elliptica & Bacillariophyceae & & & & $\mathbf{x}$ & & & & & \\
\hline Merismopedia tenuissima & Cyanophyceae & $x$ & & & & & & & & \\
\hline Navicula erifuga & Bacillariophyceae & & $x$ & & & & & & & \\
\hline Navicula placentula & Bacillariophyceae & & & & $\mathbf{x}$ & & & & & \\
\hline Navicula sigmoides & Bacillariophyceae & & & & $x$ & & & & & \\
\hline Navicula sp & Bacillariophyceae & $\mathrm{X}$ & & & & & & & & \\
\hline Nitzschia acicularis & Bacillariophyceae & $x$ & & & & & & & & \\
\hline Nitzschia longissima & Nitzschia & & $x$ & & & & & & & \\
\hline Nitzschia sp. & Bacillariophyceae & $x$ & & & & & & & & \\
\hline Peridinium umbonatum & Dinophyceae & & $x$ & & & & & & & \\
\hline Pinnularia cardinalis & Bacillariophyceae & $\mathbf{x}$ & $x$ & & $x$ & & & & & \\
\hline Pleurosigma sp & Bacillariophyceae & $x$ & & & & & & & & \\
\hline Scenedesmus acumunatus & Chlorophyceae & $x$ & & & & & & & & \\
\hline Sirrurella sp & Bacillariophyceae & & $\mathbf{x}$ & & $x$ & & & & & \\
\hline Spirogyra sp & Zygnematophyceae & & & & $x$ & & & & & \\
\hline Strombomonas fluviatilis & Euglenophyceae & $\mathbf{x}$ & & & & & & & & \\
\hline
\end{tabular}

$\mathrm{Sm}=$ Sarotherodon melanotheron, $\mathrm{Tg}=$ Tilapia guineensis; $\mathrm{Hf}=$ Hemichromis fasciatus; $\mathrm{Hb}=$ Hemichromis bimaculatus $\mathrm{Gm}=$ groupe des mulets (Mugil cephalus, M. curema; Liza grandisquamis, L. bananensis; L. falcipinis); $\mathrm{Pj}=$ Pomadasys jubelini; El = Elops lacerta $; \mathrm{Ta}=$ Thorogobius angolensis; $\mathrm{Ef}=$ Ethmalosa fimbriata

Le facteur qualitatif relatif à la présence ou à l'absence du phytoplancton dans le suc stomacal des poissons a permis d'examiner le régime alimentaire des poissons dans les étangs. Une analyse factorielle de correspondance (Figure 12) a mis en évidence des similarités de régime alimentaire entre les poissons. 
Graphique symétrique (axes F1 et F2: 41,03\%)

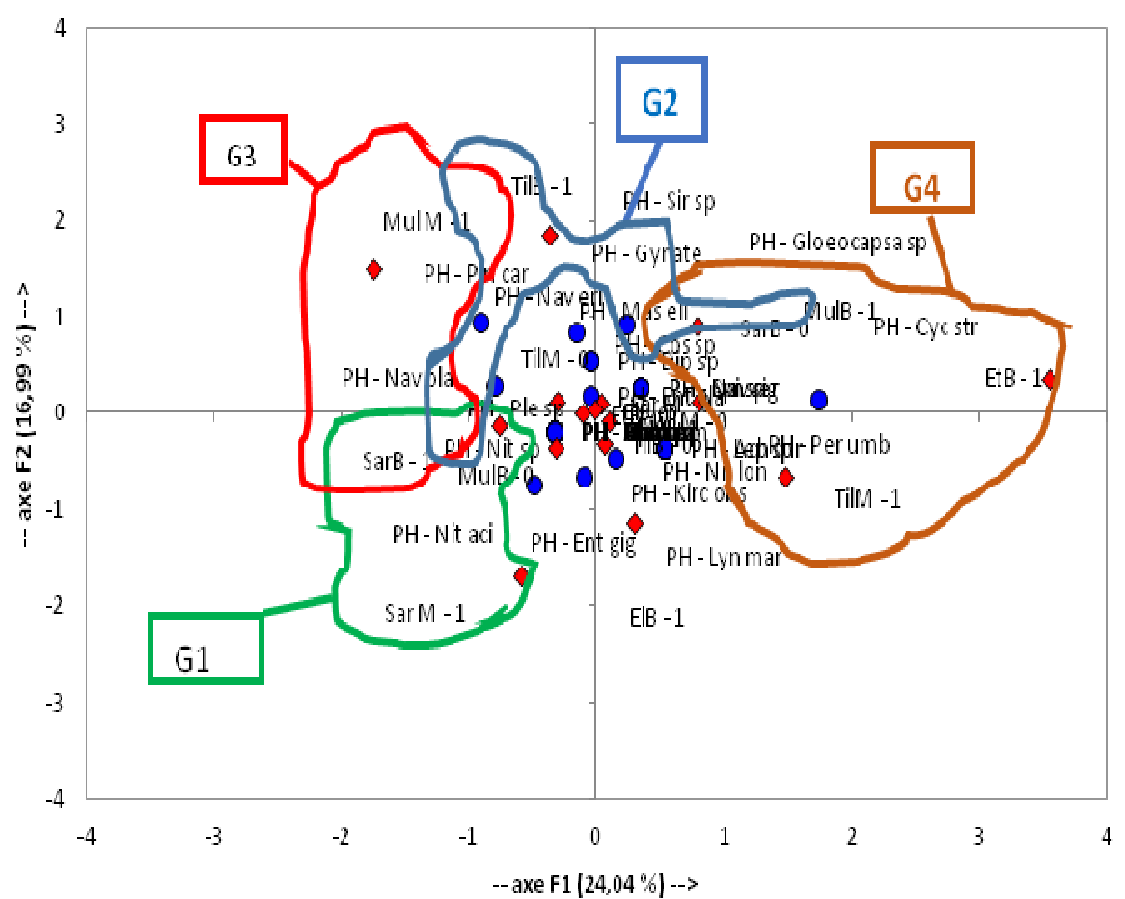

Figure 12 : Régime alimentaire des poissons sur la base d'une analyse factorielle de correspondance (AFC).

Cette AFC montre que le groupe 1 est constitué d'individus de Sarotherodon melanotheron ayant le même régime alimentaire aussi bien à Bandial qu'à Mlomp. Dans ces deux contrées, l'espèce se nourrit en partie de Nitzschia acicularis. Le groupe 2 est constitué d'individus de Sarotherodon melanotheron, de Tilapia guineensis et de mulets qui se nourrissent en partie de Gyrosigma atenuatum. Le groupe 3 est constitué d'individus de Tilapia guineensis de Bandial, de Sarotherodon melanotheron de Bandial et des mulets de Mlomp qui se nourrissent aussi de Pinnularia cardinalis. Le groupe 4 est constitué d'individus de Ethmalosa fimbriata de Bandial, de Tilapia guineensis de Mlomp et des mulets de Bandial qui se nourrissent tous de Cyclotella striata. Les espèces dont le centre d'inertie est proche de l'origine des axes de l'AFC constituent celles ayant généralement un régime alimentaire mono-phytoplancton. Par ailleurs, il existe une interférence entre le groupe 1 et le groupe 3 puisque Sarotherodon melanotheron de Bandial se nourrit aussi de Pinnnularia cardinalis comme le groupe de mulets de Mlomp. II est de même entre le groupe 2 et le groupe 3 étant donné que Sarotherodon melanotheron de Bandial partage le même régime alimentaire que le groupe de mulets de Mlomp eu égard à Pinnnularia cardinalis. Une troisième interférence est notée entre le groupe 2 et le groupe 4 du fait que les mulets et Ethmalosa fimbriata de Bandial consomme Cyclotella Striata au même titre que Tilapia guineensis à Mlomp. Le partage des aliments noté témoigne de la plasticité du régime alimentaire planctophage des poissons dans la zone. Par ailleurs l'analyse du contenu stomacal des poissons a aussi révélé l'absence de phytoplanctons chez Hemichromis fasciatus à Bandial et la présence de détritus végétaux chez les fretins de Tilapia guineensis et chez Hemichromis bimaculatus à Bandial. Chez le groupe des mulets de Bandial, il a été noté la présence de vers de terre, de pattes d'insectes et de détritus végétaux chez les fretins de toutes les espèces confondues. Elops lacerta se nourrit de Kircheneriella obesa et de détritus dans la zone de Bandial. A Samatite le suc stomacal de Thorogobius angolensis présente aussi des détritus végétaux. Globalement, il ressort de l'étude du régime alimentaire l'existence d'un groupe des poissons planctophage (phytoplancton, diatomées...) et d'un groupe détritivore (boue, colloïdes, matière organique...) Cependant, le régime alimentaire est dès fois fonction de l'âge ou de la taille certaines espèces comme c'est le cas chez les fretins de Tilapia guineensis et du groupe des mulets qui sont détritivores tout en présentant parfois des indices de régime alimentaire carnivore marqué par la 


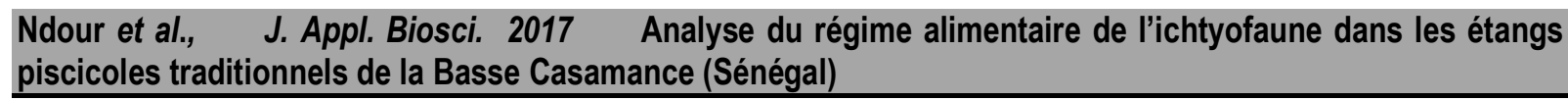

présence de vers de terre ou de restes d'insectes. Ce résultat laisse entrevoir un régime alimentaire omnivore chez les fretins de ces poissons.

Perception du régime alimentaire des poissons par les pisciculteurs de la zone d'étude : Les résultats de l'enquête sur la nourriture des poissons dans les étangs montrent que $98,6 \%$ des producteurs connaissent leurs aliments dans la zone (Tableau 4).

Tableau 4 : Aliments cités dans l'alimentation des poissons dans la zone

\begin{tabular}{|l|l|l|l|l|l|}
\hline Aliments des poissons & Bandial & Mlomp & Aliments des poissons & Bandial & Mlomp \\
\hline Boue de mangrove & $\mathbf{+}$ & $\mathbf{+}$ & Phytoplancton & $\mathbf{+}$ & $\mathbf{+}$ \\
\hline Feuilles décomposées de palétuviers & $\mathbf{+}$ & $\mathbf{+}$ & Plants de riz & & $\mathbf{+}$ \\
\hline Bouses ou lisiers de vache & $\mathbf{+}$ & $\mathbf{+}$ & Déchets ou fèces d'oiseaux & $\mathbf{+}$ & \\
\hline Herbes aquatiques & $\mathbf{+}$ & $\mathbf{+}$ & Matière organique et détritus & $\mathbf{+}$ & \\
\hline Propagules de palétuviers & & $\mathbf{+}$ & Déchets d'animaux (mangouste) & $\mathbf{+}$ & \\
\hline Vers de terre & & $\mathbf{+}$ & Paille de riz (résidus des rizières) & & $\mathbf{+}$ \\
\hline Algues & $\mathbf{+}$ & $\mathbf{+}$ & Les restes des repas & $\mathbf{+}$ & \\
\hline Diaspores de Avicennia germinans & $\mathbf{+}$ & $\mathbf{+}$ & Larves et insectes aquatiques & $\mathbf{+}$ & \\
\hline Racines de palétuviers & $\mathbf{+}$ & $\mathbf{+}$ & Prédation entres espèces & & $\mathbf{+}$ \\
\hline Son de riz & $\mathbf{+}$ & & & & \\
\hline
\end{tabular}

Au total, 19 aliments sont cités par les producteurs de la zone. $73,68 \%$ de ces aliments font partie du régime alimentaire des poissons selon les producteurs de Bandial contre $68,42 \%$ selon ceux de Mlomp. Les aliments cités à la fois dans les deux contrées sont au nombre de huit et ceux cités par l'une ou l'autre sont aussi notés (tableau 4). La différence, de diversité des aliments, notée dans la zone montre que le régime alimentaire des poissons, est connu en partie des pisciculteurs. Cette bonne connaissance, des aliments, fonde leur à ne pas apporter de la nourriture aux poissons élevés. Par ailleurs, l'observation des poissons dans les étangs a permis d'identifier des herbacées leur servant de nourriture (Figure 13). A Mlomp, deux cypéracées font partie du régime alimentaire des poissons. II s'agit de Cyperus esculentus dont les fleurs, en décomposition sont consommées par Tilapia guineensis. Les feuilles et des fleurs de Bolboschoenus maritimus en décomposition sont aussi consommées par Tilapia guineensis. Sporobolus virginicus est la troisième espèce consommée par Tilapia guineensis et Hemichromis bimaculatus. Le poisson, dont le régime alimentaire est omnivore (détritivore et carnivore) est Hemichromis fasciatus qui se nourrit d'insectes et de larves parfois à la suite de sauts acrobatiques spectaculaires pour capturer les insectes qui voltigent au-dessus des eaux.

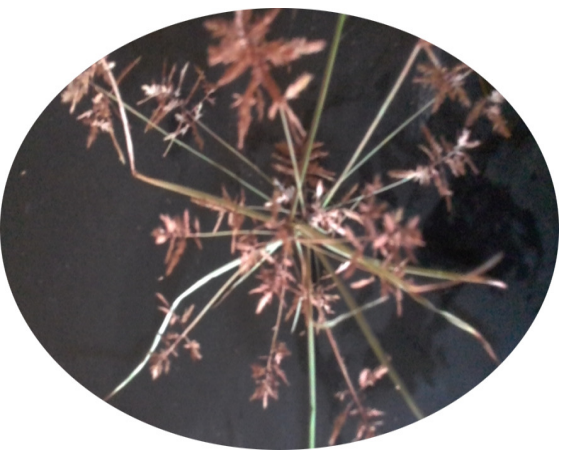

Cyperus esculentus

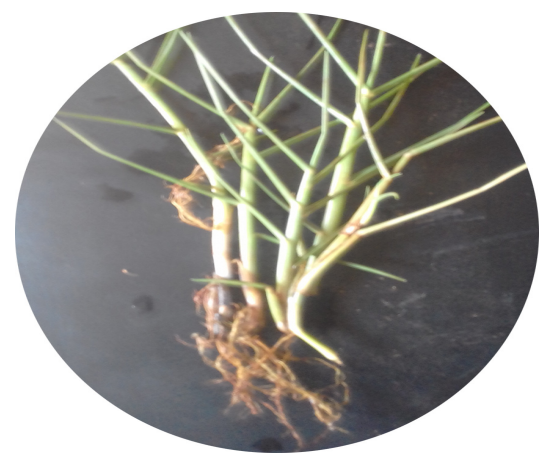

Sporobolus virginicus

Figure 13 : Herbacées consommées par les poissons 


\section{DISCUSSION DES RESULTATS}

Diversité des poissons dans les étangs piscicoles : La diversité des poissons est plus importante à Bandial (16 espèces) qu'à Mlomp (15 espèces). Fondamentalement, la différence relève de la présence où de l'absence de poissons d'intérêt commercial dans les deux contrées. A cet égard, il ressort que les espèces d'importance économique non négligeable sont plus présentes à Bandial qu'à Mlomp. Parmi les espèces d'importance économique, on note: Trachinotus teraia, Sphyraena barracuda Solea senegalensis et Pomadasys jubelini à Bandial. Malheureusement, aucune de ces espèces de poissons n'atteint la taille commerciale à la fin d'un cycle d'élevage. La raison de cette faible croissance biométrique des poissons serait liée à l'abandon des étangs de profondeur et au manque de main d'œuvre pour l'entretien des grandes digues des étangs (idée Casamance, 2007). Au plan socioéconomique, la diversité des espèces de poisson ne peut influencer l'engouement à la pratique de l'aquaculture ni la relance de la pisciculture traditionnelle. Néanmoins, certaines espèces de poisson atteignent des tailles intéressantes pour les producteurs à la fin d'un cycle d'élevage. II s'agit de Elops lacerta, Hemichromis fasciatus et du groupe des mulets particulièrement Mugil cephalus et Liza grandisquamis. Au regard de la diversité des poissons, la pisciculture traditionnelle demeure encore un système d'exploitation rentable en Basse-Casamance. Cependant, II reste à améliorer le régime alimentaire des poissons et l'emplacement des étangs afin de relever les défis relatifs à la qualité des poissons d'intérêt économique. Toutefois, la contrainte difficile à contourner serait le temps de croissance normale de certaines espèces de poisson avant d'atteindre la taille commerciale puisque dans les étangs, les conditions environnementales se détériorent dès la fin de la saison des pluies (Ndour et al. 2017).

Abondance et diversité du phytoplancton dans les étangs : L'abondance du phytoplancton varie en fonction des étangs et des contrées. Les étangs de Bandial ( 94 phytoplanctons) sont plus diversifiés que ceux de Mlomp (44). Ces deux contrées ont en commun 29 phytoplanctons correspondant à $30,15 \%$ des effectifs de Bandial et $65,9 \%$ de ceux de Mlomp. L'explication serait liée à la proximité $(2,5 \mathrm{~m})$ entre les étangs et les habitations dans la zone de Bandial particulièrement entre les étangs et les porcheries à Eloubalir. Les porcheries seraient donc de potentielles sources de microorganismes pour les étangs piscicoles de Bandial. Ce résultat confirment ceux de Hem et al. (2001) en guinée Conakry où l'évacuation des déchets de porcherie dans les étangs a accrue la concentration en chlorophylle passant de $0,7 \mathrm{mg} / \mathrm{l}$ à $6,4 \mathrm{mg} / \mathrm{l}$ dans les enclos.

Régime alimentaire des poissons dans les étangs piscicoles: En Basse Casamance, les pisciculteurs s'attachent à la capacité des étangs à nourrir les poissons élevés. Cette logique est la même au Niger où les populations comptent sur la production naturelle du milieu en aliments (CNEDD, 2001) L'analyse du régime alimentaire des poissons sur la base d'une AFC a montré l'existence d'une alimentation variée dans les étangs. II y'a 3 grands groupes de poissons dont le groupe qui ne consomme pas le phytoplancton, celui qui en consomme 2 à 3 espèces à la fois et le groupe de ceux qui n'en consomment qu'une seule espèce de phytoplancton. Cependant, le groupe 2 est constitué de poissons qui partagent plusieurs phytoplanctons dans le réseau trophique. Cette diversité de régime alimentaire des poissons dans les systèmes d'élevage multi-trophiques a été démontrée sur les habitudes alimentaires des poissons-chats dans le bassin de la rivière Lefini au congo (Moutsinga et al, 2012). A cet égard, certains phytoplanctons notamment Ntizschia sp.Gyrosigma atenuatum, Pinnularia cardinalis, et Cyclotella striata retrouvées dans le contenus stomacal des poissons élevés en Basse-Casamance, l'ont été aussi chez les Citharius par Lausanne (1988). La diversité de régime alimentaire que dénotent les cinq groupes issus de l'AFC montre l'existence d'une plasticité alimentaire qui permet aux poissons de s'adapter au mieux aux conditions environnementales des étangs. Cet avantage explique certainement la taille d'environ $50 \mathrm{~cm}$ de longueur atteinte par certaines espèces de poissons comme Mugil cephalus, Liza grandisquamis et Elops lacerta. Chez Elops lacerta, l'analyse du suc stomacal n'a pas permis de montrer un régime alimentaire tel que évoqué dans le lac Nokoué par Villanueva (2004). Toutefois, l'espèce se nourrit du phytoplancton Kircheneriella obesa. Chez les mulets, le régime alimentaire est constitué de Diatomées telles que Ntzschia sp. Gyrossigma atenuatum, Mastogloia elliptica, ces résultats confirment ceux de Sarr et al. (2013) qui indiquent que ce régime est essentiellement, composé de diatomées, de débris organiques et de grains de sable. Ce régime alimentaire a aussi été décrit chez le mulet Mugil curema par Sène (1997) et UICN (2017). Chez Ethmalosa fimbriata et Pomadasys jubelini, les sujets n'atteignent pas la taille commerciale ce qui pourrait s'expliquer par la faible diversité de leur régime alimentaire et du partage des phytoplanctons avec les autres espèces de poissons. Toutefois, le régime alimentaire identifié chez Pomadasys 
jubelini confirme la présence de larves de Chironomidae et de restes d'insectes dans le suc stomacal de spécimens capturés dans la lagune Ebrié en Côte d'Ivoire (Koné et al, 2007). Chez Sarotherodon melanotheron et Tilapia guineensis, l'étude montre que leur régime alimentaire est planctophage, ce qui confirme leur régime alimentaire composé de phanérogames, d'algues vertes, d'algues rouges, d'algues bleues, d'algues brunes, de diatomées selon Kidé et al. (2015). Ce régime alimentaire herbivore est marqué par une plus grande consommation de phytoplancton chez Tilapia guineensis dans la zone. D'ailleurs, Ndour et al. (2001) affirme que Tilapia guineensis dispose d'un spectre trophique plus large et tend à consommer plus de vase (bivalves) que de débris végétaux, lorsque la salinité augmente. Ce résultat confirme le régime alimentaire planctophage et détritivore à partir de la pellicule de fond des étangs riche en algues sédimentées selon Ndiaye (2004). Cet argument fonde de plus en plus la pratique de l'élevage de poissons herbivores, ce qui simplifie l'alimentation tout en diminuant les impacts sur la nature. Cette option milite en faveur de la pisciculture traditionnelle à l'image d'un système intégré où les différentes cultures pourront interagir entre elles en diminuant l'impact des déchets produits par le système sur l'environnement puisque ce type d'interaction leur permet d'optimiser l'utilisation de l'espace, des nutriments et d'améliorer les rendements (Hussenot et al. 2004). En résumé, l'étude du régime alimentaire des poissons dans les étangs a révélé deux chaînes alimentaires: une planctonique et une détritique.

\section{CONCLUSION}

L'étude du régime alimentaire des poissons élevés en Basse Casamance a permis de relever un réseau trophique composé d'une chaîne alimentaire planctophage et d'une autre détritivore. La famille des Cichlideae constituée de Tilapia guineensis et Sarotherodon melanotheron présente la plus large gamme de planctons consommés dans la première chaine. Tilapia guineensis, Hemichromis bimaculatus et Thorogobius angolensis font partie des poisons présentant un régime alimentaire détritivore dans certains étangs. Un régime alimentaire omnivore a été noté chez Hemichromis fasciatus et Pomadasys jubelini qui se nourrissent de détritus végétaux, de larves et d'insectes dans les étangs. Parmi les aliments notés, il y'a des herbacées aquatiques comme Cyperus esculentus dont
Ce genre de réseau trophique a été décrit dans le Lac Tchad où le premier niveau trophique, de la chaîne planctonique, était constitué de végétaux particulièrement de phytoplanctons et la deuxième d'une pellicule organique des fonds du lac (Lauzane, 1972).

Perception du régime alimentaire des poissons par les producteurs: Les aliments des poissons sont connus des pisciculteurs à cause de leurs savoirs empiriques sur l'aquaculture mais aussi grâce à quelques formations ou innovations (apport de nourriture) réalisées par des institutions comme l'Agence nationale de l'aquaculture (ANA), la mission vietnamienne et idée Casamance (Ndiaye, 2004). Les connaissances livrées par les producteurs corroborent les résultats issus de l'analyse du suc stomacal des poissons issus des étangs. Des débris végétaux, des pattes d'insectes, de la boue de mangrove, du sable etc. ont été rencontrés dans les sucs digestifs des poissons notamment chez Hemichromis fasciatus, Hemichromis bimaculatus et Pomadasys jubelini. Des vers de terre ont été aussi retrouvés dans les contenus stomacaux des fretins du groupe des mulets. La boue de mangrove comme aliment des poissons confirme les résultats d'études antérieures qui montrent que c'est la pellicule d'aspect floconneux formée d'une phase détritique (débris végétaux fins, algues...) et d'une phase organique vivante (bactéries, diatomées benthiques, protozoaires...) qui motivent l'ingestion de la couche superficielle du sol par certains poissons (Lauzane, 1972)

la matière en décomposition sert de nourriture à Tilapia guineensis. Entre autres herbacées, il y a Bolboschoemus maritimus et Sporobolus virginicus dont la biomasse aérienne en décomposition est consommée par Tilapia guineensis et Hemichromis bimaculatus. La nourriture disponible dans les étangs permet donc aux pisciculteurs de produire des poissons de qualité. $\mathrm{Ce}$ Savoir local a permis de se lancer dans la fabrication d'un aliment pour les poissons dans les élevages traditionnels. Ainsi, un apport alimentaire pourrait permettre d'arriver à une production spectaculaire et de qualité chez les principales espèces de poissons. En tout état de cause, la diversité de régie alimentaire des poissons et la valorisation du savoir local devrait aboutir au développement la pisciculture traditionnelle. 


\section{Ndour et al., J. Appl. Biosci. 2017 Analyse du régime alimentaire de l'ichtyofaune dans les étangs}

piscicoles traditionnels de la Basse Casamance (Sénégal)

\section{REMERCIEMENTS}

Les auteurs adressent leurs remerciements au Laboratoire de botanique et de biodiversité végétale de l'Université Cheikh Anta Diop de Dakar qui a assuré

\section{RÉFÉRENCE BIBLIOGRAPHIQUES}

ANSD, 2015. Situation économique et sociale, 2012. Rapport régional de Ziguinchor, août, 2015, $7 \mathrm{p}$.

Bambara AJD, 1989. Étude d'un système traditionnel d'exploitation du milieu: Cas des bassins piscicoles de la Basse Casamance (Sénégal), École inter -États des sciences et médecine vétérinaires de Dakar, 80p.

Bassene OA. 2012. L'évolution des mangroves de la Basse Casamance au Sud du Sénégal au cours des 60 dernières années : surexploitation des ressources, pression urbaine, et tentatives de mise en place d'une gestion durable. Géographie; Université de Lyon, 2016. INNT : 2016 LYSES040.

Bassene OA. Bassene OA., Cubizolle H, Cormier-Salem MC, BA SY. 2013. L'impact des changements démographiques et socio-économiques sur la perception et la gestion de la mangrove en Basse Casamance (Sénégal), revue. Org., vol. 88/4 | 2013 pp297-2015. DOI : 10.4000/geocarrefour.9306 ISSN : 1960-601X.

CNEDD, 2001. Expérience du NIGER en Matière de pêche et de Pisciculture, Projet NER/98/001/NEX, PNUD /PNEDD, 29 p.'

Hem S, Curtis MI, Sène MA, Sagbla C, 2001. Pisciculture Extensive en Guinée Forestière Modèle de développement intégré et rizipisciculture, rapport final du projet 7 .Aep.GUl.104 -Convention CEE/IRD, $85 \mathrm{p}$.

Hussenot J, 2004. Les systèmes intégrés en aquaculture marine : une solution durable pour un meilleur respect de l'environnement littoral, actes du Colloque Pêche et Aquaculture : pour une exploitation durable des ressources vivantes de la mer et du littoral, Nantes, 21-23 janvier 2004, Université de Nantes-Géolittomer, 11p. Rev Ivoir. Sci. Technol., 25(2015)188-203., ISSN 1813-3290, http://www.revist.ci., consulté le $11 / 08 / 2017$

Idée Casamance, 2007. La revalorisation des bassins piscicoles traditionnels en Casamance, www.ideecasamance.org, 17p. Consulté le 13 février 2017.

Kidé NG, Dia M, Yarba L, KONE Y, Khalil F, Salhi G; Bouksir H, Saoudy Y, 2015. Écologie trophique l'analyse des eaux des étangs et du bol alimentaire des poissons sur une durée relativement longue.

de Sarotherodon melanotheron heudeleutii et de Tilapia guineensis (Perciformes; Ciclidea) Du parc National du Banc d'Arguin, Mauritanie, Rev. Ivoir. Sci. Technol., 25 (2015) 188.

Koné T, Kouamelan EP, Ouattara NI, Kicho AV, 2007. Régime alimentaire de Pomadasys jubelini (Pisces, Haemulidae) dans une lagune Ouest africaine (lagune Ebrié, Côte d'Ivoire) dans Sciences \& Nature Vol. 4 No1 : $65-73$ (2007).

Lauzanne L, 1988. Régimes alimentaires des principales espèces de poissons de l'archipel oriental du lac Tchad, ORSTOM, pp18-23.

Lauzanne L, 1972. Régimes alimentaires des principales espèces de poissons de l'archipel oriental du lac Tchad, Verh. Internat. Verein. Limnol.18 636 646 Stuttgart, Novembre, 1972.

AN Moutsinga, C Ngokaka, F Akouango, V Mamonekene 2012. Diversité Du Régime Alimentaire Des Poissons-chats du Bassin De la Rivière Lefini (Congo) En Fonction Des Saisons, Journal Home, vol 24, №2.

Ndiaye V, 2004. Comment améliorer les bassins piscicoles traditionnels de Kagnout, Bessire, Kartiack et Thiok Essyl Situés dans les zones humides en Casamance, Sénégal, idée Casamance, $14 \mathrm{p}$.

Ndour N, Sambou B, Diadhiou A, Sambou, Dasylva M, 2017. Atouts et contraintes de la pisciculture traditionnelle de Bandial (Casamance, Sénégal) in J. Biol. Che. Sci., Internationale formulate group. All right reserved (sous presse): http://www.ifgdg.org.

Ndour I; Le loch F, Thiaw TO, Ecoutin JM, LAË, JR, Sadio O, De Morais TL, 2011. Étude du régime alimentaire de deux espèces de Cichlidae en situation contrastée dans un estuaire tropical inverse d'Afrique de l'Ouest (Casamance, Sénégal); Sci. Halieut. Aquat, 4 120-133. Mis en ligne le 30/12/2011. Oceanraise () 150811-29.

Sène $N, 1997$. Contribution à l'étude qualitative du régime alimentaire de quelques poissons dans l'estuaire du Sine-Saloum (Sénégal); Centre de recherche océanographique de Dakar, $40 \mathrm{p}$.

Bassene, OA, Cubizolle H, Cormier-Salem MC et Sy BA, 2013. «L'impact des changements 
démographiques et socio-économiques sur la perception et la gestion de la mangrove en Basse Casamance (Sénégal) », Géocarrefour [En ligne], vol. 88/4 | 2013. URL: http://geocarrefour.revues.org/930, DOl: 10.4000/geocarrefour.9306, consulté le 10 août 2017.

Sarr SM, Kabre JA, T Niass F, 2013. Régime alimentaire du mulet jaune (Mugil cephalus, Linneaus, 1758, Mugilidea) dans l'estuaire du fleuve Sénégal, Journal of Applied Biosciences 71:5663- 5672, ISSN 1997-5902. URL : http://www.m.elewa.org/JABS/2013/71/1.pdf consulté ce 21/08/2017.

UICN, 2017.he UICN red list of threatened species; ISSN 2307-8235 (c) International Union for Conservation of Nature and Natural Resources, $5 p$. http://www.iucnredlist.org/ pdf consulté ce 10/11/2017.

VILLANUEVA S M C.2004. Biodiversité et relations trophiques dans quelques milieux estuariens et lagunaires de l'Afrique de l'Ouest : Adaptations aux pressions environnementales, doctorat de I'Institut National Polytechnique de Toulouse, $272 \mathrm{p}$. 